\title{
The Next-generation Biomass for Biorefining
}

\author{
Hah Young Yoo ${ }^{a}$ and Seung Wook Kim ${ }^{\text {b,* }}$
}

\begin{abstract}
Biorefining, which uses biomass as feedstock and converts it into valuable products, is a core technology for the sustainable green industry and has high potential as an alternative to the current petrochemical-based industry. This article covers the requirements for feedstock that should be met for the economic feasibility of a biorefinery. In particular, organic waste that meets several requirements as the next-generation biomass has high potential. However, the complex and significant differences in composition depending on the origin make it difficult to follow the previous system of classification such as cellulose, hemicellulose, and lignin. In particular, several organic wastes contain high value-added bioactive components. Therefore, a strategy for the effective use of high value-added ingredients contained in trace amounts is required, which is briefly introduced in the third section of this article.
\end{abstract}

Keywords: Biomass; Biorefinery; Pretreatment; Saccharification; Fermentation

Contact information: a: Department of Biotechnology, Sangmyung University, 20, Hongjimun 2-Gil, Jongno-Gu, Seoul 03016, Republic of Korea; b: Department of Chemical and Biological Engineering, Korea University, 145, Anam-Ro, Seongbuk-Gu, Seoul 02841, Republic of Korea;

*Corresponding author: kimsw@korea.ac.kr

\section{Biomass Classification for Biorefining}

Biorefining is a concept derived from the refining of petrochemical resources. It is defined as the production of biofuels and biomaterials from biomass based on biotechnology (Yang et al. 2015; Ibrahim et al. 2018). In particular, a biorefinery, which uses carbon-neutral biomass as raw material and converts it into useful materials, is attracting great attention as an alternative to the existing petroleum-based refining because it is a core technology of a sustainable green industry (Kim et al. 2018, 2020).

In general, biorefining technology is divided into three generations according to the feedstock of biomass. First-generation biomass utilization, which relies on food crops such as corn, sugar cane, wheat, and potatoes, is in the commercialization stage. However, such processing has been recognized as a threat to food security, including the rising of grain or feed prices and resulting starvation of underserved people (Kline et al. 2016). Second-generation biofuels, by contrast, are derived from plant-based material composed of cellulose, hemicellulose, and lignin, which are the most abundant renewable resources on earth. However, the potential of the feedstock is relatively low, as it contains about 15 to $25 \%$ lignin, which cannot be used in fermentation. In particular, a pretreatment process should be additionally performed before the saccharification, which is one of the biggest barriers for commercialization because it accounts for about 30 to $40 \%$ of the total process cost (Kim et al. 2018; Siripong et al. 2018). In order to overcome the shortcomings of these resources, genetically engineered crops or algae (without lignin components) are classified as third-generation biomass, which merits accelerated commercialization (Lee et al. 2019; Sa et al. 2021). However, additional land and costs are incurred for cultivation, and algae require a higher level of extraction and conversion technology for utilization. 
Therefore, the main factors that will determine the economic feasibility for nextgeneration biomass are proposed to be as follows:

1) Cost and availability: Ideally, feedstock should be inexpensive, have consistent quality, and year-round availability at centralized locations.

2) Content of active ingredient per unit weight: It should contain sufficient nutrients required for microbial culture to be used for fermentation.

3) The levels and range of impurities: There should be no constituents affecting the fermentation and purification steps.

4) It should be easy to handle and low transportation and storage costs.

5) Denaturation problems in saccharification or sterilization processes: The ingredients should not be denatured during the preparation of the medium.

\section{The Next-generation Biomass as Organic Waste from Bioindustries}

Based on the development of genetic engineering, it is possible to bioconvert target bio-products using various organic wastes as feedstock (Kim et al. 2020). The utilization of organic wastes such as agricultural residues, crude glycerol, fruits peel, juice processing residues, municipal wastes, spent coffee grounds, waste cooking oils, and waste paper sludge has been reported, and its origins are very diverse: urban, agricultural, food processing, paper industry, and biodiesel production processes (Ibrahim et al. 2018; Estrada-Martínez et al. 2019; Lee et al. 2020a,b). These organic wastes have various advantages such as no required farmland, low price, and year-round availability. In particular, such resources have been recognized as attractive feedstock for biorefinery due to the effects that can be obtained while preventing environmental pollution. Now the possibility of organic waste is being carefully reviewed as fourth-generation biomass (Yang et al. 2015).

There are a variety of organic wastes that can be obtained easily and inexpensively; however, the composition is closely related to their origin. To achieve effective utilization of biomass, it often has been classified into cellulose, hemicellulose, and lignin depending on the solid composition. However, in the case of organic waste, the content of other components can be relatively high or valuable, making it difficult to follow the previous classification system. For example, spent coffee grounds are composed of cellulose, galacto-mannan, lipids, protein, lignin and other ingredients, the content of which depends on the type of bean. Citrus peel comprises about $25 \%$ of the total weight of the fruit and is composed of cellulose, pectins, essential oils, phenols, vitamins, carotenoids, hesperidin, and limonin. In particular, several organic wastes have been reported to contain high valueadded bioactive ingredients; thus, strategies for effective utilization of the components should be suggested.

\section{Application Strategy for Next-generation Biomass}

Organic wastes derived from food processing residues, such as fruit peels, coffee grounds, and juice residues, contain bioactive ingredients. In order to utilize the biomass more effectively, the concept of the food processing residue biorefinery consisting of four steps is shown in Fig. 1. The first step is to collect the biomass and perform an analysis of their composition, including trace amounts of bioactive ingredients. Through this process, strategies for extraction of valuable substances contained in samples, design of biological 
processes, improvement in conversion efficiency, and potential of resources can be evaluated. The second step is to select an effective extraction method for target bioactive ingredients such as carotenoids, flavonoids, polyphenols, and vitamins contained in the sample and to optimize extraction conditions. The value of the resource can be improved through the extraction of bioactive ingredients. In addition, removal of the high-value components can be expected to facilitate sugar conversion during enzymatic hydrolysis by weakening the binding of carbohydrates contained in the solid fraction (Choi et al. 2020). The third step is the conversion of carbohydrates such as cellulose and hemicellulose in the solid fraction to fermentable sugars. Enzymatic conversion can be significantly improved through the selection of an appropriate pretreatment and optimal conditions. The hydrolysates from the saccharification can be used as the medium for microbial fermentation in the next step. The final step is the bioconversion process such as fermentation, fabrication, or synthesis. The bioactive ingredients extracted in the previous step can be applied to produce functional polymers such as bioelastomers (Lee et al. 2020a). The hydrolysate containing fermentable sugars can be utilized in the production of platform chemicals such as butanediol and propanediol as well as biofuels such as ethanol and butanol by microbial fermentation (Ibrahim et al. 2018; Siripong et al. 2018; Lee et al. 2020b). Finally, the by-products from each conversion process can be converted to biochar through pyrolysis, and a zero-waste process could be achieved by reusing by-products as feedstock.

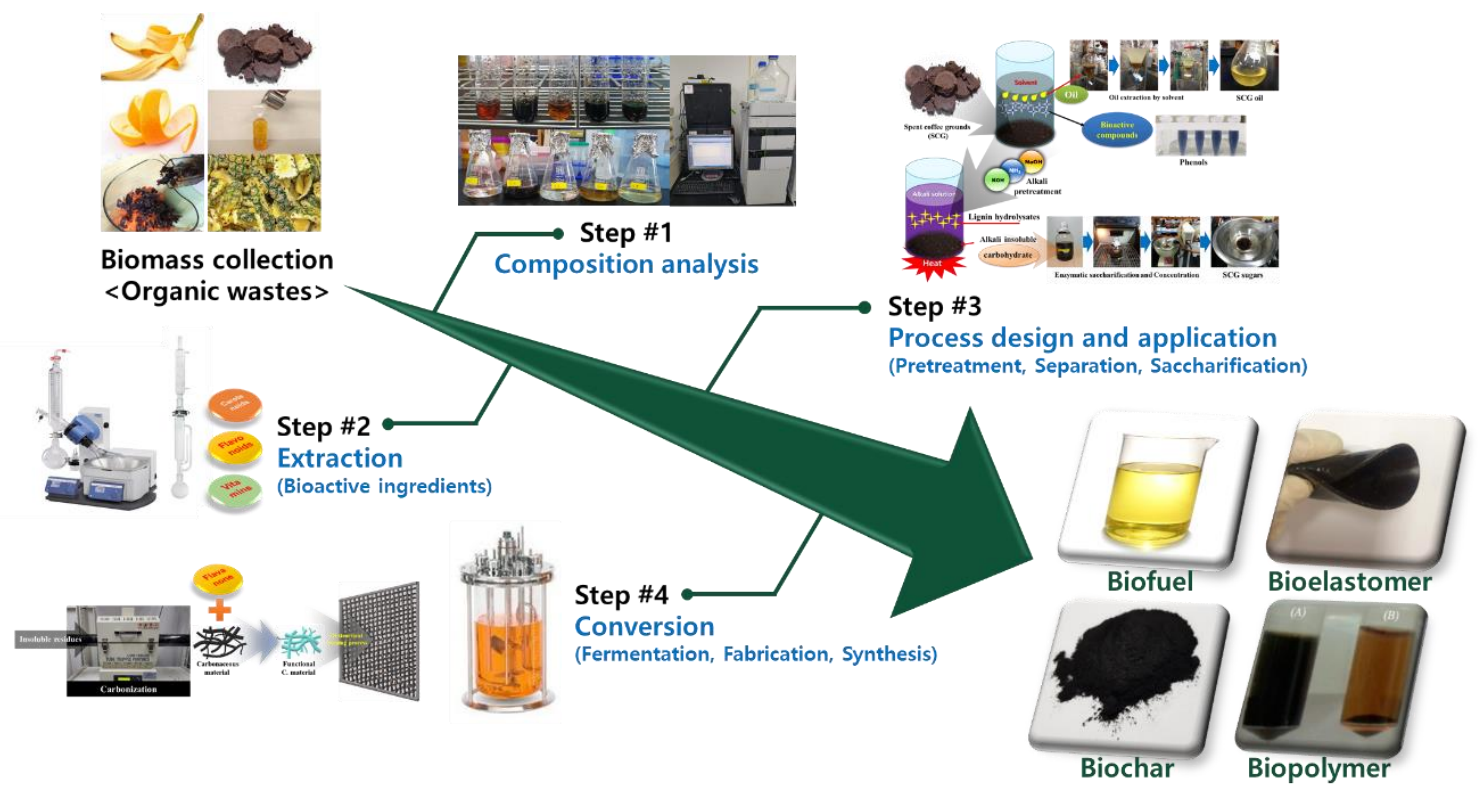

Fig. 1. Concept of food processing residue biorefinery

\section{Acknowledgments}

This work was supported by the National Research Foundation of Korea (NRF) grant funded by the Ministry of Science and ICT (MSIT) (NRF-2019R1A2C1006793 and NRF-2020R1C1C1005060). 


\section{References Cited}

Choi, M., Kang, Y. R., Zu, H. D., Lim, I. S., Jung, S. K., and Chang, Y. H. (2020). "Effects of time on phenolics and in vitro bioactivity in autoclave extraction of raviola (Annona muricata) leaf," Biotechnol. Bioproc. E. 25(1), 9-15. DOI: 10.1007/s12257-019-0259-3

Estrada-Martínez, R., Favela-Torres, E., Soto-Cruz, N. O., Escalona-Buendía, H. B., and Saucedo-Castañeda, G. (2019). "A mild thermal pre-treatment of the organic fraction of municipal wastes allows high ethanol production by direct solid-state fermentation," Biotechnol. Bioproc. E. 24(2), 401-412. DOI: 10.1007/s12257-019-0032-7

Ibrahim, M. F., Kim, S. W., and Abd-Aziz, S. (2018). "Advanced bioprocessing strategies for biobutanol production from biomass," Renew. Sust. Energ. Rev. 91, 1192-1204. DOI: 10.1016/j.rser.2018.04.060

Kim, C. K., Choi, H. S., Lee, S. J., Lee, J. H., Lee, J. H., Yoo, H. Y., Han, S. O., and Kim, S. W. (2018). "Production of xylanase from a novel engineered Pichia pastoris and application to enzymatic hydrolysis process for biorefinery," Process Biochem. 65, 130-135. DOI: 10.1016/j.procbio.2017.11.001

Kim, S. Y., Yang, Y. H. and Choi, K. Y. (2020). "Bioconversion of plant hydrolysate biomass into biofuels using an engineered Bacillus subtilis and Escherichia coli mixed-whole cell biotransformation," Biotechnol. Bioproc. E. 25, 477-484. DOI: 10.1007/s12257-019-0487-6

Kline, K. L., Masangi, S. M., Dale, V. H., Woods, J., Souza, G. M., Osseweijer, P., Clancy, J. S., Hilbert, J. A., Johnson, F. X., McDonnell, P. C. and Mugera, H. K. (2016). "Reconciling food security and bioenergy: priorities for action," $G C B$ Bioenergy 9, 557-576. DOI: 10.1111/gcbb.12366

Lee, J. H., Lee, H. U., Lee, J. H., Lee, S. K., Yoo, H. Y., Park, C., and Kim, S. W. (2019). "Continuous production of bioethanol using microalgal sugars extracted from Nannochloropsis gaditana," Korean J. Chem. Eng. 36(1), 71-76. DOI: 10.1007/s11814-018-0173-y

Lee, K. H., Chun, Y., Jang, Y. W., Lee, S. K., Kim, H. R., Lee, J. H., Kim, S. W., Park, C., and Yoo, H. Y. (2020a). "Fabrication of functional bioelastomer for food packaging from Aronia (Aronia melanocarpa) juice processing by-products," Foods 9(11), 1565. DOI: 10.3390/foods9111565

Lee, J. H., Yoo, H. Y., Lee, S. K., Chun, Y., Kim, H. R., Bankeeree, W., Lotrakul, P., Punnapayak, H., Prasongsuk, S., and Kim, S. W. (2020b). "Significant impact of casein hydrolysate to overcome the low consumption of glycerol by Klebsiella aerogenes ATCC 29007 and its application to bioethanol production," Energ. Convers. Manage. 221, 113181. DOI: 10.1016/j.enconman.2020.113181

Sa, M., Zhang, B., and Zhu, S. (2021). "Miscanthus: Beyond its use as an energy crop," BioResources 16(1), 5-8.

Siripong, P., Doungporn, P., Yoo, H. Y., and Kim, S. W. (2018). "Improvement of sugar recovery from Sida acuta (Thailand Weed) by $\mathrm{NaOH}$ pretreatment and application to bioethanol production," Korean J. Chem. Eng. 35(12), 2413-2420. DOI: 10.1007/s11814-018-0170-1

Yang, X., Choi, H. S., Park, C., and Kim, S. W. (2015). "Current states and prospects of organic waste utilization for biorefineries," Renew. Sust. Energ. Rev. 49, 335-349.

DOI: 10.1016/j.rser.2015.04.114 